\title{
The Microbiological Context of HIV Resistance: Vaginal Microbiota and Mucosal Inflammation at the Viral Point of Entry
}

\author{
John J. Schellenberg' ${ }^{1}$ and Francis A. Plummer ${ }^{1,2,3}$ \\ ${ }^{1}$ Department of Medical Microbiology, Faculty of Medicine, University of Manitoba, 260-727 McDermot Avenue, \\ Winnipeg, MB, Canada R3E 3P5 \\ ${ }^{2}$ National Microbiology Laboratory, Canadian Science Centre for Human and Animal Health, 1015 Arlington Street, \\ Winnipeg, MB, Canada R3E 3R2 \\ ${ }^{3}$ Department of Medical Microbiology, University of Nairobi, P.O. Box 30197-00100, Nairobi, Kenya
}

Correspondence should be addressed to John J. Schellenberg, schellej@cc.umanitoba.ca

Received 13 November 2011; Accepted 4 January 2012

Academic Editor: Yingzi Cong

Copyright (c) 2012 J. J. Schellenberg and F. A. Plummer. This is an open access article distributed under the Creative Commons Attribution License, which permits unrestricted use, distribution, and reproduction in any medium, provided the original work is properly cited.

\begin{abstract}
Immune activation is increasingly recognized as a critical element of HIV infection and pathogenesis, causing expansion of virus founder populations at the mucosal port of entry and eventual exhaustion of cellular immune effectors. HIV susceptibility is well known to be influenced by concurrent sexually transmitted infections; however, the role of commensal vaginal microbiota is poorly characterized. Bacterial vaginosis (BV) is a risk factor for HIV acquisition in studies worldwide; however, the etiology of BV remains enigmatic, and the mechanisms by which BV increases HIV susceptibility are not fully defined. A model of how vaginal microbiota influences HIV transmission is considered in the context of a well-established cohort of HIV-exposed seronegative (HESN) commercial sex workers (CSW) in Nairobi, Kenya, many of whom have increased levels of anti-inflammatory factors in vaginal secretions and reduced peripheral immune activation (immune quiescence). Elucidation of the relationship between complex microbial communities and inflammatory mucosal responses underlying HIV infection should be a priority for future prevention-focussed research.
\end{abstract}

\section{Introduction}

Fatal opportunistic infections associated with what is now known as the acquired immunodeficiency syndrome (AIDS) were first described in 1981 among young, previously healthy homosexual men in the United States [1]. While global media and health authorities were focussed on stigmatized "risk groups" in North America, largely heterosexuallytransmitted epidemics were already well established throughout sub-Saharan Africa by the time the human immunodeficiency virus (HIV) was discovered in 1983. A cohort-based study of commercial sex workers (CSW) in Nairobi, Kenya found that HIV prevalence increased from $4 \%$ to over $60 \%$ between 1981 and 1985 [2]. Follow-up work identified a subgroup of CSW who were never infected with HIV despite years of exposure, one of the earliest described examples of highly HIV-exposed seronegative (HESN) individuals in the world [3].
HIV infection results in profound, multifactorial immune dysregulation eventually leading to AIDS usually after a period of 7-10 years. This lengthy asymptomatic period is characteristic of the lentiviridae family of viruses to which HIV and a wide variety of simian immunodeficiency viruses (SIV) belong. Progressive destruction of CD4+ T helper (Th) cell populations via direct viral killing is generally believed to explain slow disease progression; however, recent studies in nonhuman primates have revealed that critical events in the earliest phases of infection determine SIV/HIV immunopathogenesis-a "mucosal catastrophe" [4-7].

The purposes of this review are to describe dynamics of mucosal inflammation as critical determinants of HIV transmission and AIDS pathogenesis, influences of vaginal microbiota as the specific "microbiological context" in which HIV transmission occurs, and how these factors may 
interact to block HIV transmission in highly HIV-exposed seronegative (HESN) commercial sex workers from Nairobi, Kenya.

\section{The Nature of HIV Resistance}

Although rarely reported in non-human primates challenged with SIV [8], individuals in diverse groups worldwide appear to resist HIV infection despite repeated exposure. This variability in susceptibility to HIV infection and/or its pathogenic consequences has been intensively studied and found to correlate with a variety of viral, genetic, immunological, and sociobehavioural variables; however, the precise mechanisms of protection in HESN individuals have not been elucidated. Study of this phenomenon is hampered by lack of a clear terminology or definition of who should or should not be classified as resistant to HIV, difficulties in comparing groups of individuals, and the small numbers of individuals included in these studies $[9,10]$.

Results from some studies indicate that cell-mediated responses to HIV, possibly subsequent to an aborted infection, may result in protection against the establishment of HIV systemically [11]. Late seroconversion of HESN individuals with preexisting HIV-specific CTL responses, possibly following a break from CSW, indicates that ongoing exposure to CSW and/or HIV may be required to maintain the resistant phenotype [12]. Intriguingly, HIV resistance has also been observed in mothers and sisters of HESN cohort members [11]. Single nucleotide polymorphisms (SNP) in noncoding regions of the interferon regulatory factor- (IRF-) 1 gene in HESN CSW correlate with reduced expression of IRF-1 [13]. Among other effects, reduced IRF1 production may lead to generalized hyporesponsiveness to proinflammatory stimuli mediated by IFN $\gamma$. Generally lower levels of basal gene transcription and downregulation of proinflammatory cytokines in Th from HESN CSW suggest a "quiescent" immune profile [14]. Reduced activation of Th/CTL and increased frequency of Tregs in HESN compared to other HIV - individuals indicates that increased Treg may potently downregulate Th activation levels [15].

Screening of cervicovaginal lavage (CVL) protein samples from individuals in the Majengo cohort revealed increased concentrations of the anti-inflammatory serine protease inhibitor trappin-2 (also known as elafin) in CVL from HESN compared to other HIV - and HIV+ individuals [16]. Recently, the epithelial origin and suspected antiviral properties of this molecule were confirmed, with studies showing direct inactivation of HIV particles during coincubation with trappin-2 [17]. Further proteomic studies have identified upregulation of several other antiproteases in vaginal fluid of HESN individuals, including serine protease inhibitors and cystatin, a known anti-HIV factor [18].

Multiple and often conflicting immunological influences in systemic and mucosal compartments have been investigated to gain insight into the HESN phenotype. Although little consensus has emerged, the "immune quiescence" hypothesis offers a plausible biological explanation for why viral populations at points of entry do not engender productive infections in HESN individuals.

\section{Mechanisms of Vaginal HIV Transmission}

The efficiency of male-to-female sexual transmission is very low, in the range of one productive infection for every 200-2000 exposures [19], demonstrating the formidable physicochemical and immunological barrier of the female genital tract (FGT) (Figure 1(a)). Semen from infected individuals, especially those with bacterial coinfections, is known to contain very high concentrations of free virus as well as large numbers of infected lymphocytes and monocytes that may directly transfer infectious virus to vaginal epithelium, T-helper (Th) or antigen-presenting cells such as macrophages (Mo), and Langerhans/dendritic cells (LC/DC) at the mucosal point of entry (Figure 1(b)). The first barrier that the virus must cross is the mucus layer, a dense multilayered network of glycoprotein molecules (mucins) colonized by high concentrations of diverse microorganisms (vaginal microbiota). As well as creating a dense fluid gel overlying the epithelium, mucins bind covalently to epithelial cells to create a tightly adherent layer called the glycocalyx [20]. Both cell-free and cell-associated virus may become trapped in these networks and subsequently inactivated by low $\mathrm{pH}$ and hydrogen peroxide $\left(\mathrm{H}_{2} \mathrm{O}_{2}\right)$ originating from specific types of bacteria, or by epithelialderived proteins with antiviral properties, such as defensins or secretory leukocyte protease inhibitor (SLPI) and related effectors [7, 21]. Even under artificial infection conditions with a very high inoculum of pure virus, only a tiny proportion of virions gain access to susceptible cells in the epithelium and LP [7], with productive infection frequently initiated by a single viral particle [22]. Changes in the amount and consistency of excreted mucus, the profile and activity of colonizing microbiota, the amount and nature of epithelial defence molecules due to phase of menstrual cycle, sexual intercourse, coinfections, stress, and nutrition are all likely to influence the likelihood that HIV can cross this initial physical barrier.

Several mechanisms for crossing the stratified squamous epithelium of the vagina and ectocervix or the simple columnar epithelium of the endocervix and upper reproductive tract to access target cells have been proposed; however, the relative importance of each during infection in vivo has not been defined. Both HIV and SIV enter target cells in the FGT primarily after binding the CD4 cell-surface glycoprotein (receptor) and the chemokine ligands CCR5 or CXCR4 (coreceptors) found on target cells throughout FGT epithelial surfaces and lamina propria (LP); however, distribution of various immune cell types has been shown to vary greatly between individuals and anatomical sites, and is strongly influenced by mucosal inflammation [23]. Normal vaginal epithelium is characterized by intraepithelial CD8+ cytotoxic lymphocytes (CTL), relatively few Th restricted to the LP, and low numbers of LC in basal epithelial layers. However, greatly increased intraepithelial Th, and LC are observed in women with chronic vaginal inflammation [23]. The highest concentrations of Mo, Th, and CTL are found associated with the "transformation zone" (TZ) of the exocervical os (Figure 1(b)), where stratified epithelium abruptly ends and the endocervical monolayer begins, indicating a critical 


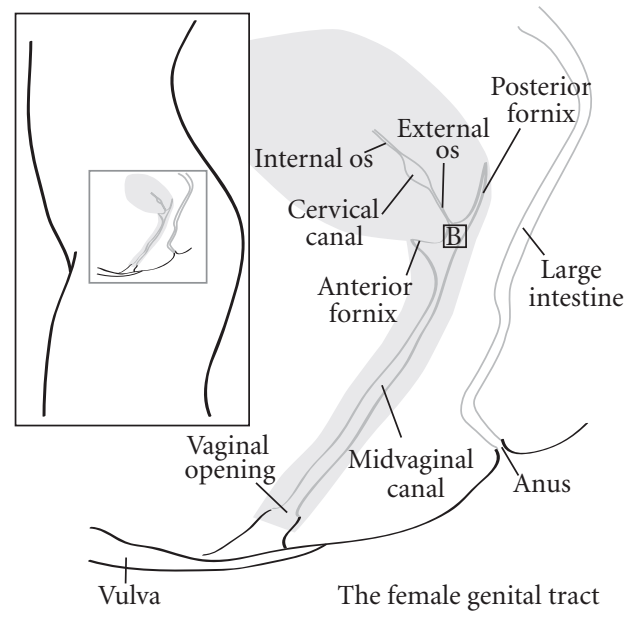

(a)
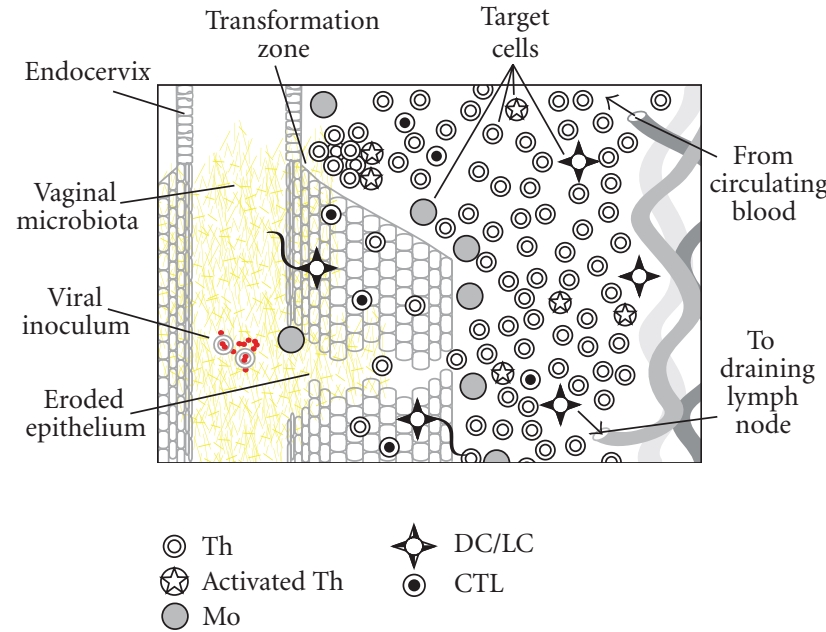

(b)

Figure 1: Mechanisms of vaginal HIV transmission. (a) Overview of the major anatomical features of the female genital tract (FGT). (b) Overview of hypothetical mechanisms of HIV transmission in the female genital tract. See text for details.

position in immune control for ascending pathogens and also increased vulnerability to HIV infection [23]. These observations suggest distinct "immune microenvironments" associated with different anatomical sites within the FGT. Although the cervix is likely to be a preferred site of entry for HIV given higher concentrations of target cells in these regions, especially in the TZ, large increases in numbers of target cells in the vaginal epithelium observed during inflammation increase vulnerability to HIV infection in these tissues as well.

Penetration of the epithelial layer has been shown to happen within minutes of exposure in experiments with non-human primates [19]. HIV binds lipid moieties such as galactosyl cerebroside ( $\mathrm{GalC}$ ) or other receptors on vaginal epithelial cells [24], leading to transcytosis across or productive infection of the epithelial layer [25]. Intraepithelial and submucosal APC such as LC and DC are thought to play an important role in viral transport to large populations of susceptible cells in the lymphatic tissue. Terminal mannose residues on HIV surface glycoproteins bind to related receptors or lectins such as langerin in intraepithelial LC [26]. Since LC form a tight network at the mucosal surface, and are known to sample the luminal space with specialized cell extensions [19], they are likely to be among the first cells to come into contact with HIV. Viral binding to langerin has been found to trigger virus degradation and block cisinfection via an endosomal pathway that is unique to LC. In the absence of activating stimuli, LC resist infection with HIV and transmit virus much less efficiently to Th when compared to submucosal DC. However, activation of LC by TLR stimulation and epithelial production of the cytokine $\mathrm{TNF} \alpha$ in the context of vaginal coinfections abolishes the protective role of langerin and facilitates cis-infection and migration of LC to lymphatic tissue [26].

Although the precise pathway travelled by HIV during the majority of sexually transmitted infections is unknown, it is clear that genital coinfections or other factors that (1) increase shedding of free virus and infected cells by the infecting partner, (2) increase permeability of the protective mucus layer, (3) cause changes in the composition and function of vaginal microbiota, (4) disrupt the physical integrity of the epithelial barrier, or (5) increase the abundance of target cells at the mucosal port of entry, will increase the likelihood that HIV will be transmitted during heterosexual intercourse.

\section{Early Tolerogenic Signals and Mucosal Catastrophe: A Shifting Paradigm}

Once founder populations of virus have adsorbed and penetrated target cells at the port of entry, there follows a period of several days in which there is little or no productive expansion of viral populations. This "eclipse phase" (Figure 2(a)) represents a critical opportunity to mitigate expansion of very small populations of virus in spatially dispersed foci amid a relatively small concentration of target cells in lamina propria (LP) relative to lymphoid tissue [7]. Inflammation and immune activation near the port of entry are likely to determine expansion or decay of HIV founder populations [27]. If the virus reproductive rate exceeds unity (i.e. each infected cell results in the infection of at least one other cell), the threshold to productive infection (the "fast phase") is crossed on or about day seven (Figure 2(b)). The virus-as-signal is amplified and broadcast distally from the port of entry in LP to draining lymph nodes, the systemic circulation, and all lymphatic tissue, rising to peak levels by day ten to fourteen [7] (Figure 2(c)). As a result of direct viral killing and Fas/FasL-mediated apoptosis in the presence of high concentrations of viral gp120, mucosal Th populations, concentrated in gut-associated lymphoid tissue (GALT), vanish almost entirely within four days $[5,7]$. This 

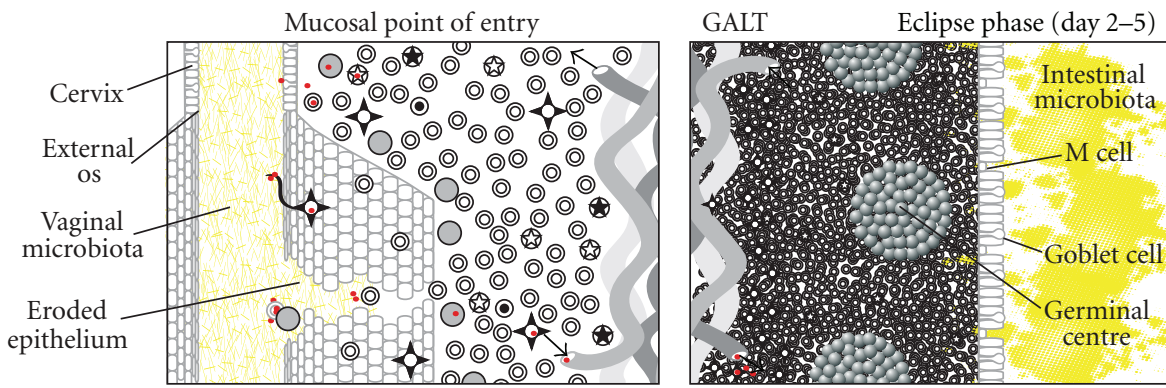

(a)
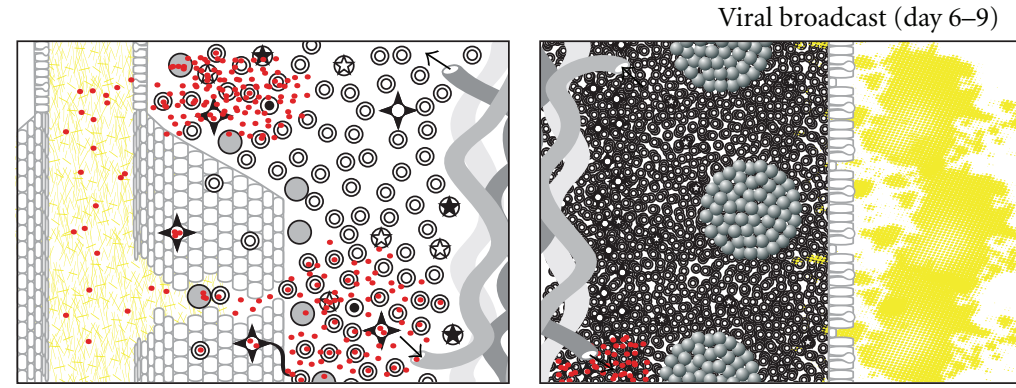

(b)

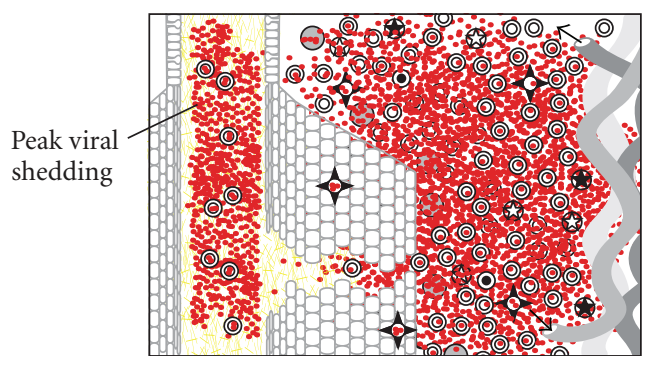

Peak viremia (day 10-14)

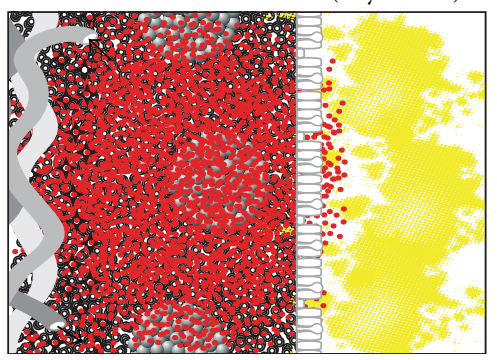

(c)

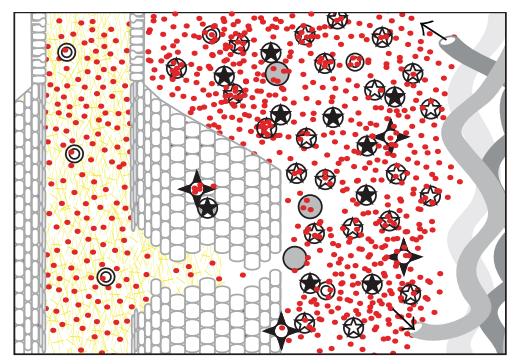

Chronic immune activation (slow phase)

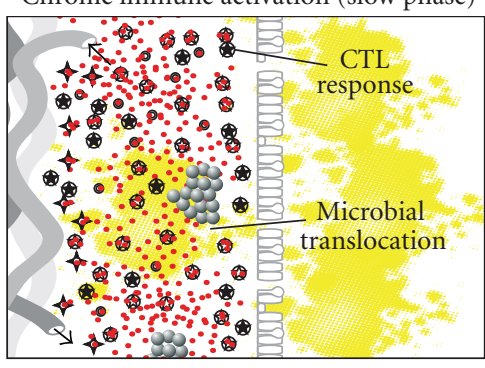

$\begin{array}{lcl}\text { Lumen Epithelium } & \text { CP } & \\ \text { (2) } \mathrm{CD} 4+\mathrm{T} & \bigcirc \text { Macrophage } \\ \text { Activated } \mathrm{CD} 4+\mathrm{T} & \$ \text { Dendritic/langerhans } \\ \text { CD8+T } & \bigcirc \text { B lymphocyte } \\ \text { Activated CD8+T } & \end{array}$

(d)

Figure 2: A mucosal catastrophe. Overview of the "fast phase" of HIV infection in the days following infection, including the eclipse phase (a), the viral broadcast phase (b), peak viremia (c), and subsequent chronic immune activation (d). See text for details. 
massive immunological insult is not reflected in the systemic circulation, where only a minority of Th reside, explaining earlier models of gradual Th depletion in disease progression based on measuring Th in peripheral blood only [7].

The "slow phase" of infection (Figure 2(d)) following the initial insult is marked by chronic, generalized immune activation, including increased activation and turnover of Th, and increased systemic proinflammatory cytokines [28]. Immune activation has been found to be a better predictor of disease progression than viral load, but whether the virus drives immune activation or vice versa is unresolved. Immune activation increases target cells for HIV infection and the self-perpetuating relationship between activation and viral replication leads to eventual clonal exhaustion of Th memory pools, as reflected in rising viral loads and declining Th counts as disease progresses [28]. Although antiretroviral therapy can mitigate immune defects by reducing viral replication, continued immune activation remains a barrier to immune reconstitution following treatment [28]. In "elite controllers," defined as HIV-infected individuals who maintain very low viral loads in the absence of treatment, progressive loss of Th correlates with immune activation [28]. Therefore, regardless of virus control in later stages of infection, chronic immune activation continues to strongly influence disease progression, likely due to translocation of immune-stimulating microbial products such as lipopolysaccharide (LPS) across damaged mucosal barriers in the gut. Consistent with this hypothesis, a recent study has found higher LPS levels in the blood of chronically HIV-infected people [29].

In contrast, many SIV, including SIVagm from African green monkeys, cause no immunopathology in their natural hosts, despite intense viral replication and massive depletion of Th in systemic and mucosal compartments during acute infection [30, 31]. Surprisingly, this initial insult to Th populations does not result in enteropathy or chronic immune activation in nonpathogenic infections, and the function of various immune cell subsets is preserved. Although Th numbers in peripheral blood and mucosal tissues rise in the weeks following acute infection by both pathogenic and nonpathogenic SIV, steep declines in the chronic phase are only observed in pathogenic SIV infection. No increases in Th activation or apoptosis are observed in chronic nonpathogenic SIV infection, while activation and proliferation of CTL occurs early and then subsides, with very little detectable LPS in serum indicating preservation of the gut barrier and absence of microbial translocation. Upregulation of inflammatory cytokines and beta chemokines is not observed in serum of SIVagm-infected green monkeys [31, 32]. Instead, upregulation of TGF- $\beta$ stimulates expression of FoxP3 in Th and CTL within one day of infection, leading to high levels of immunosuppressive IL-10 at early timepoints [32]. This early induction of the $\mathrm{T}$ regulatory (Treg) subset and IL-10 may counteract immune activation signals due to chronic infection in SIVagm-infected green monkeys.

An altered balance between Treg populations and the related Th17 subset has also been identified in pathogenic SIVmac infection of the pigtailed macaque, but not in nonpathogenic SIVagm infection of the green monkey [33].
Th17 cells express IL-17 and IL-22, cytokines that have been shown to be important for immune responses to extracellular bacteria in the gut, where Th17 cells are concentrated [34]. IL-17 induces IL- 8 to promote recruitment of neutrophils to sites of bacterial infection, while IL-22 induces proliferation of intestinal epithelial cells and repair of the mucosal barrier, as well as stimulating epithelial production of antibacterial defensins. Th17 cells and Treg are derived from a common progenitor cell and their reciprocal differentiation depends on the cytokine milieu generated by DC with TLR stimulated by bacterial products in peripheral tissues [33]. In humans, the Th17 phenotype is induced in naïve $\mathrm{T}$ cells in the presence of IL1 $\beta$ and IL-6 [35]. Interestingly, most $\alpha 4 \beta 7+$ cells in GALT of rhesus macaques have been shown to be Th17 cells [36]. Therefore, preferential depletion of Th17 cells in pathogenic SIV, as well as in HIV infection [34], may be due to the affinity of SIV/HIV gp120 for $\alpha 4 \beta 7$ integrin on gut mucosal Th, leading to permeability of mucosal barriers in the gut, microbial translocation, and chronic immune activation. The early upregulation of Treg in nonpathogenic SIV infection may contribute to balanced ratios of Th17/Treg cell populations in GALT and mitigate immune dysfunction leading to AIDS [34].

These observations strongly suggest that the timing and pattern of host immune responses to infection play a critical role in determining disease outcome. Induction of early tolerogenic responses to founder populations may block virus propagation and broadcasting into systemic compartments, or mitigate damage to mucosal Th populations thereby reducing immune activation resulting from microbial translocation. Immune activation at compromised mucosal surfaces is a critical component of both vulnerability to vaginal HIV transmission and AIDS pathogenesis in the gut; however, nothing is known about the dynamics of normal microbiota in these contexts.

\section{The Microbiological Context: A Model}

Vaginal microbiology is predicted to influence susceptibility of mucosal surfaces to HIV infection via a number of direct and indirect mechanisms (Figure 3). Genital tract infections such as chlamydia, gonorrhea, syphilis, herpes simplex virus (HSV), human papilloma virus (HPV), and trichomoniasis are known to increase the risk of HIV acquisition [37-40]. The "enigmatic" clinical entity known as bacterial vaginosis (BV) $[41,42]$, associated with increased susceptibility to many sexually transmitted infections, has been shown to increase risk for HIV infection in numerous studies from around the world [43]. BV is a frequently asymptomatic clinical condition defined by a reduction in vaginal Lactobacillus populations and overgrowth of anaerobic and Gram-negative organisms [42]. Increasingly linked with negative reproductive health outcomes, such as miscarriage, premature birth, postoperative infections, pelvic inflammatory disease, and HIV infection, its etiology and clinical course remain poorly defined with a consequent lack of effective prevention and treatment strategies [41, 44, 45].

The current "gold standard" for diagnosis of BV is based on observation of clinical markers, known as Amsel's criteria, 


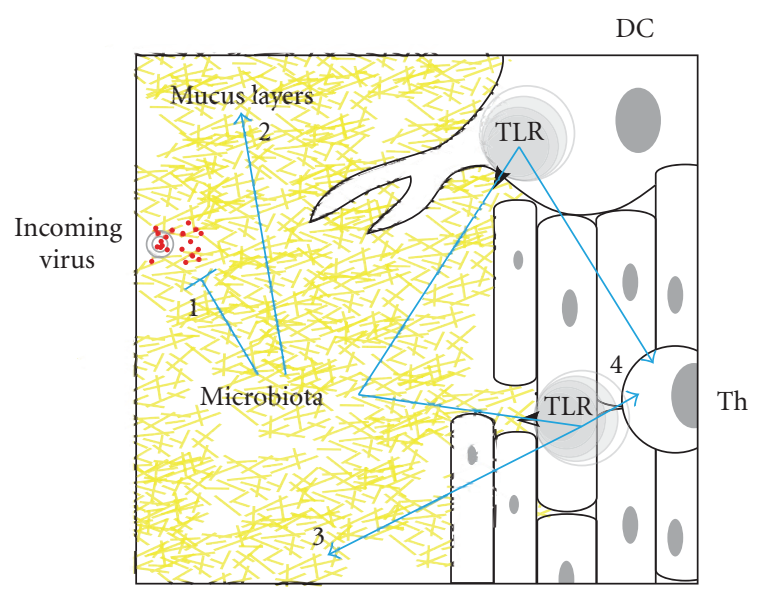

Figure 3: The microbiological context of HIV resistance. Vaginal microbiology influences susceptibility of the mucosal layer to HIV infection in multiple interrelated ways, including (1) direct effects on viral particles or infected allogenic cells via binding, mannose scavenging, or bacterial metabolites (acid, $\mathrm{H}_{2} \mathrm{O}_{2}$ ), (2) alteration of quantity/viscosity/permeability of mucin network in mucus layer via proteases, (3) stimulation of innate mucosal defence molecules and cells via TLR stimulation, and (4) modulation of T-cell activation and susceptibility via conditioning by TLR-stimulated dendritic cells, macrophages, or epithelial cells.

including thin, milky discharge, high vaginal $\mathrm{pH}$, presence of "clue cells" (shed epithelial cells covered in Gram-negative bacteria), and amine ("fishy") odour [46]. In the laboratory setting, most studies rely on scoring profile of microbial types in Gram-stained vaginal swab smears [47]. These systems are based on the relative abundance of Lactobacillus morphotypes (short to long, straight-sided rods) compared to BV bacteria morphotypes (Gram-negative/variable coccobacilli and curved rods).

Quantitative bacteriology and microbial ecology are of particular concern in understanding BV, which is defined by changes in levels of total bacteria and alterations in abundance of bacterial subpopulations, rather than the presence of a specific pathogen [44]. For example, BV-associated bacteria such as Gardnerella, Mobiluncus, Atopobium, and Mycoplasma species are routinely isolated from women without BV [45]. Simultaneous isolation of Mycoplasma, Gardnerella, and anaerobes, described as a "pathologic core," combined with failure to isolate Lactobacillus in a vaginal sample, has been found to be strongly associated with BV [48]. Despite a general lack of external signs of inflammation in BV (as indicated by the -osis suffix signifying overgrowth, rather than the -itis suffix signifying inflammation) [49], many studies associate BV with increased proinflammatory signalling via TLR stimulation [50-52]. BV is characterized by an increase in the overall bacterial load in the vagina, with possible implications for TLR stimulation and immune activation. The presence of various proteolytic enzymes during BV demonstrate the importance of mucin lysis as a source of energy for the bacterial consortium [53], and may also explain nutritional synergies observed in culturebased experiments with BV bacteria [54]. Enzymes such as sialidase and prolidase have been shown to impair innate and adaptive immune effectors in the vagina, including cytokines, immunoglobulins, and cellular receptors, and are likely to explain lack of overt signs of inflammation despite local production of pro-inflammatory signals during BV [55]. Thinning of the vaginal mucus layer may also increase opportunities for HIV to access target cells in underlying mucosa.

Given a preeminent ecological position in the vaginal environment [56], colonization of the female genital tract by Lactobacillus species is increasingly recognized as critical for overall vaginal health and resistance to infection by bacterial and viral pathogens, including HIV [57]. Under the influence of estrogen, vaginal epithelial cells store glycogen, which is hydrolysed to glucose and metabolized by vaginal bacteria [58]. Excreted lactate, especially by Lactobacillus species, reduces the overall $\mathrm{pH}$ of the vaginal lumen to "normal" levels, in the range of 3.9 to $4.5[59,60]$. Along with effectors such as $\mathrm{H}_{2} \mathrm{O}_{2}$ and bacteriocins, acid production by Lactobacillus is believed to discourage overgrowth of other bacterial genera including Streptococcus, Gardnerella, Bacteroides, and Mycoplasma [61] $\mathrm{H}_{2} \mathrm{O}_{2}$ produced by a Lactobacillus strain has been shown to inactivate HIV in vitro [62]. Production of acid and $\mathrm{H}_{2} \mathrm{O}_{2}$ may be synergistic, since bacterial $\mathrm{H}_{2} \mathrm{O}_{2}$ is more likely to remain stable at lower $\mathrm{pH}$ in vitro [63]. The ability of Lactobacillus to produce acid from mannose scavenged as a carbon source has also been hypothesized to block HIV infection through digestion of mannosylated residues on viral glycoproteins [64]. Interestingly, two promising molecules currently being studied as potential topical agents for blocking HIV infection (microbicides) also bind mannose residues on the viral surface $[65,66]$.

Absence of culturable $\mathrm{H}_{2} \mathrm{O}_{2}$-producing Lactobacillus has been shown to be an important contributing factor in elevated risk for cervicitis observed in those with BV [67]. This finding confirms that BV-related organisms are associated with an elevated risk of genital tract inflammation, the converse of which may be a non/anti-inflammatory role for Lactobacillus organisms. The possibility of antiinflammatory effects by Lactobacillus organisms in the vagina is all but unexplored, although several studies concerning the gut epithelium indicate induction of epithelial defence molecules, anti-inflammatory signalling, and increased Treg by specific strains of Lactobacillus [55, 68-70].

We recently conducted the first study to address BV and Lactobacillus colonization specifically as possible factors contributing to HIV resistance in highly exposed commercial sex workers fron Nairobi [71]. Compared to other HIVindividuals, HESN in the Majengo cohort were just as likely to be diagnosed with $\mathrm{BV}$ and had similar vaginal $\mathrm{pH}$ in a cross-sectional study, a finding confirmed by retrospective analysis of BV diagnoses over several years in nearly 1,000 individuals [71]. Ultradeep molecular characterization of vaginal microbiota also revealed few differences in species distribution between HESN and other HIV - individuals [72]; however, neither study was conclusive. BV is known to induce a pro-inflammatory environment and create vulnerability to HIV, so the ability of an individual to 
modulate some aspect of the inflammatory process related to the BV cycle may determine her ability to block viral infection. Longitudinal studies examining dynamics of vaginal microbiota and mucosal responses over time will be necessary in order to definitively address the potential impact of vaginal microbiology on HIV resistance in the Majengo cohort.

\section{Conclusions}

The immune activation hypothesis of HIV pathogenesis suggests that multiple factors may contribute to reduced viral broadcast at the mucosal port of entry, either blocking productive infection entirely or limiting damage to lymphoid tissue subsequent to peak viremia. Therefore, these phenomena may be seen as being part of a continuum of HIV resistance [10]. HESN CSW in the Majengo cohort are among the most striking and well-studied examples of HIV resistance worldwide. Ongoing characterization of this group has revealed many immunogenetic factors that may contribute to relative resistance in a subgroup of CSW, including recent findings indicating a role for reduced T-cell activation or "immune quiescence." Mucosal studies have revealed the possibility that HESN CSW may have increased anti-inflammatory factors in mucosal fluids, mirroring reduced immune activation in peripheral blood. Together, these studies suggest a unified set of factors in HESN women that reduce susceptibility of target cells in vivo or hamper viral expansion from the mucosal port of entry.

Although the vaginal microbiota has so far not been found to play a critical role in HIV resistance, the purpose of this review has been to clarify the role that mucosal inflammation and microbiological dynamics play in the earliest stages of HIV disease. In natural infections, the best predictor of immunodeficiency and disease is not lentiviral replication within the host but T-cell activation and activation-induced cell death leading to depletion of critical cell populations. Early immunosuppressive signals and upregulation of $\mathrm{T}$ regulatory cells within 24 hours of infection are characteristic of nonpathogenic infections, despite subsequent intense viral replication and initial depletion of CD4 in blood and lymphoid tissue in both pathogenic and nonpathogenic infections, while CSW who resist HIV infection entirely for long periods appear to have reduced levels of $\mathrm{T}$ cell activation and increased anti-inflammatory molecules in vaginal secretions.

These findings indicate that modulating immune activation, rather than directly interfering with virus, may be an alternative strategy for stopping HIV infection or mitigating pathogenesis. Whether the purposeful application of live microorganisms (probiotics) might be beneficial for these purposes has only begun to explored [73], but similar principles guide the development of HIV microbicides, defined as topical agents applied vaginally to block HIV transmission [74]. Few studies have comprehensively assessed the impact of microbicides on vaginal microbiota, examining only very limited culture-based parameters. In-depth analysis of the microbiological context is likely to be critical for assessment of candidate microbicides on composition, structure, and function of vaginal microbial communities. A concerted effort to generate detailed microbiological profiles and link this information to mucosal immune parameters will inform upcoming clinical trials for "second generation" microbicides, including probiotics, as potentially effective tools for HIV prevention.

Central questions of HIV prevention-related research in coming years might be how do microbiological and host factors interact with each other as part of the normal functioning of mucosal surfaces exposed to a wide variety of endogenous and exogenous stimuli? How do these interactions influence the likelihood that HIV introduced at the interface of host and microbiota will cross formidable physicochemical barriers and irreversibly alter host immune function? Many challenges remain to adequately define vaginal microbial community dynamics across individuals and over time.

\section{Funding}

Funding was provided by National Institutes of Health, Bill \& Melinda Gates Foundation, Canadian Institutes of Health Research (CIHR).

\section{Acknowledgment}

The authors would like to thank the women of the Majengo cohort whose ongoing commitment has made this work possible, as well as all the scientists, laboratory, clinical and administrative personnel in Nairobi and Winnipeg.

\section{References}

[1] M. S. Gottlieb, R. Schroff, and H. M. Schanker, "Pneumocystis carinii pneumonia and mucosal candidiasis in previously healthy homosexual men. Evidence of a new acquired cellular immunodeficiency," New England Journal of Medicine, vol. 305, no. 24, pp. 1425-1431, 1981.

[2] J. K. Kreiss, D. Koech, and F. A. Plummer, "AIDS virus infection in Nairobi prostitutes. Spread of the epidemic to East Africa," New England Journal of Medicine, vol. 314, no. 7, pp. 414-418, 1986.

[3] K. R. Fowke, N. J. D. Nagelkerke, J. Kimani et al., "Resistance to HIV-1 infection among persistently seronegative prostitutes in Nairobi, Kenya," Lancet, vol. 348, no. 9038, pp. 1347-1351, 1996.

[4] J. M. Brenchley and D. C. Douek, "The mucosal barrier and immune activation in HIV pathogenesis," Current Opinion in HIV and AIDS, vol. 3, no. 3, pp. 356-361, 2008.

[5] J. M. Brenchley, D. A. Price, and D. C. Douek, "HIV disease: fallout from a mucosal catastrophe?" Nature Immunology, vol. 7, no. 3, pp. 235-239, 2006.

[6] A. T. Haase, "The pathogenesis of sexual mucosal transmission and early stages of infection: obstacles and a narrow window of opportunity for prevention," AIDS, vol. 15, supplement 1 , pp. S10-S11, 2001.

[7] A. T. Haase, "Perils at mucosal front lines for HIV and SIV and their hosts," Nature Reviews Immunology, vol. 5, no. 10, pp. 783-792, 2005. 
[8] B. Peng, R. Voltan, L. Lim et al., "Rhesus macaque resistance to mucosal simian immunodeficiency virus infection is associated with a postentry block in viral replication," Journal of Virology, vol. 76, no. 12, pp. 6016-6026, 2002.

[9] R. E. Horton, P. J. McLaren, K. Fowke, J. Kimani, and T. B. Ball, "Cohorts for the study of HIV-1-exposed but uninfected individuals: benefits and limitations," Journal of Infectious Diseases, vol. 202, supplement 3, pp. S377-S381, 2010.

[10] M. Marmor, K. Hertzmark, S. M. Thomas, P. N. Halkitis, and M. Vogler, "Resistance to HIV Infection," Journal of Urban Health, vol. 83, no. 1, pp. 5-17, 2006.

[11] F. A. Plummer, T. B. Ball, J. Kimani, and K. R. Fowke, "Resistance to HIV-1 infection among highly exposed sex workers in Nairobi: what mediates protection and why does it develop?" Immunology Letters, vol. 66, no. 1-3, pp. 27-34, 1999.

[12] R. Kaul, S. L. Rowland-Jones, J. Kimani et al., "Late seroconversion in HIV-resistant Nairobi prostitutes despite preexisting HIV-specific CD8+ responses," Journal of Clinical Investigation, vol. 107, no. 3, pp. 341-349, 2001.

[13] T. B. Ball, H. Ji, J. Kimani et al., "Polymorphisms in IRF-1 associated with resistance to HIV-1 infection in highly exposed uninfected Kenyan sex workers," AIDS, vol. 21, no. 9, pp. 1091-1101, 2007.

[14] P. J. McLaren, T. B. Ball, C. Wachihi et al., "HIV-exposed seronegative commercial sex workers show a quiescent phenotype in the CD4+ T cell compartment and reduced expression of HIV-dependent host factors," Journal of Infectious Diseases, vol. 202, no. 3, pp. S339-S344, 2010.

[15] C. M. Card, P. J. McLaren, C. Wachihi, J. Kimani, F. A. Plummer, and K. R. Fowke, "Decreased immune activation in resistance to HIV-1 infection is associated with an elevated frequency of $\mathrm{CD} 4{ }^{+} \mathrm{CD} 25^{+} \mathrm{FOXP} 3^{+}$Regulatory T Cells," Journal of Infectious Diseases, vol. 199, no. 9, pp. 1318-1322, 2009.

[16] S. M. Iqbal, T. B. Ball, P. Levinson et al., "Elevated elafin/trappin-2 in the female genital tract is associated with protection against HIV acquisition," AIDS, vol. 23, no. 13, pp. 1669-1677, 2009.

[17] M. Ghosh, Z. Shen, J. V. Fahey, S. Cu-Uvin, K. Mayer, and C. R. Wira, "Trappin-2/Elafin: a novel innate anti-human immunodeficiency virus-1 molecule of the human female reproductive tract," Immunology, vol. 129, no. 2, pp. 207-219, 2010.

[18] A. Burgener, J. Boutilier, C. Wachihi et al., "Identification of differentially expressed proteins in the cervical mucosa of HIV-1-resistant sex workers," Journal of Proteome Research, vol. 7, no. 10, pp. 4446-4454, 2008.

[19] F. Hladik and M. J. McElrath, "Setting the stage: host invasion by HIV," Nature Reviews Immunology, vol. 8, no. 6, pp. 447457, 2008.

[20] M. Wilson, Microbial Inhabitants of Humans: Their Ecology and Role in Health and Disease, Cambridge University Press, Cambridge, UK, 2005.

[21] S. S. Olmsted, K. V. Khanna, E. M. Ng et al., "Low pH immobilizes and kills human leukocytes and prevents transmission of cell-associated HIV in a mouse model," BMC Infectious Diseases, vol. 5, article no. 79, 2005.

[22] J. A. Levy, "HIV pathogenesis: 25 years of progress and persistent challenges," AIDS, vol. 23, no. 2, pp. 147-160, 2009.

[23] J. Pudney, A. J. Quayle, and D. J. Anderson, "Immunological microenvironments in the human vagina and cervix: mediators of cellular immunity are concentrated in the cervical transformation zone," Biology of Reproduction, vol. 73, no. 6, pp. 1253-1263, 2005.
[24] Y. Furuta, K. Eriksson, B. Svennerholm et al., "Infection of vaginal and colonic epithelial cells by the human immunodeficiency virus type 1 is neutralized by antibodies raised against conserved epitopes in the envelope glycoprotein gp120," Proceedings of the National Academy of Sciences of the United States of America, vol. 91, no. 26, pp. 12559-12563, 1994.

[25] J. Zheng, Y. Xie, R. Campbell et al., "gp120-independent HIV infection of cells derived from the female reproductive tract, brain, and colon," Journal of Acquired Immune Deficiency Syndromes, vol. 43, no. 2, pp. 127-136, 2006.

[26] M. A. W. P. De Jong and T. B. H. Geijtenbeek, "Human immunodeficiency virus- 1 acquisition in genital mucosa: langerhans cells as key-players," Journal of Internal Medicine, vol. 265, no. 1, pp. 18-28, 2009.

[27] R. Kaul, C. Pettengell, P. M. Sheth et al., "The genital tract immune milieu: an important determinant of HIV susceptibility and secondary transmission," Journal of Reproductive Immunology, vol. 77, no. 1, pp. 32-40, 2008.

[28] D. C. Douek, M. Roederer, and R. A. Koup, "Emerging concepts in the immunopathogenesis of AIDS," Annual Review of Medicine, vol. 60, pp. 471-484, 2009.

[29] J. M. Brenchley, D. A. Price, T. W. Schacker et al., "Microbial translocation is a cause of systemic immune activation in chronic HIV infection," Nature Medicine, vol. 12, no. 12, pp. 1365-1371, 2006.

[30] A. S. Liovat, B. Jacquelin, M. J. Ploquin, F. Barré-Sinoussi, and M. C. Müller-Trutwin, "African non human primates infected by SIV-why don't they get sick? Lessons from studies on the early phase of non-pathogenic SIV infection," Current HIV Research, vol. 7, no. 1, pp. 39-50, 2009.

[31] I. Pandrea, D. L. Sodora, G. Silvestri, and C. Apetrei, "Into the wild: simian immunodeficiency virus (SIV) infection in natural hosts," Trends in Immunology, vol. 29, no. 9, pp. 419428, 2008.

[32] C. Kornfeld, M. J. Y. Ploquin, I. Pandrea et al., "Antiinflammatory profiles during primary SIV infection in African green monkeys are associated with protection against AIDS," Journal of Clinical Investigation, vol. 115, no. 4, pp. 1082-1091, 2005.

[33] D. Favre, S. Lederer, B. Kanwar et al., "Critical loss of the balance between Th17 and T regulatory cell populations in pathogenic SIV infection," PLoS Pathogens, vol. 5, no. 2, Article ID e1000295, 2009.

[34] J. M. Brenchley, M. Paiardini, K. S. Knox et al., "Differential Th17 CD4 T-cell depletion in pathogenic and nonpathogenic lentiviral infections," Blood, vol. 112, no. 7, pp. 2826-2835, 2008.

[35] E. V. Acosta-Rodriguez, G. Napolitani, A. Lanzavecchia, and F. Sallusto, "Interleukins $1 \beta$ and 6 but not transforming growth factor- $\beta$ are essential for the differentiation of interleukin 17producing human T helper cells," Nature Immunology, vol. 8, no. 9, pp. 942-949, 2007.

[36] M. Kader, X. Wang, M. Piatak et al., " $\alpha 4^{+} \beta 7^{h i} \mathrm{CD} 4^{+}$memory $\mathrm{T}$ cells harbor most Th-17 cells and are preferentially infected during acute SIV infection," Mucosal Immunology, vol. 2, no. 5, pp. 439-449, 2009.

[37] K. T. Bernstein, J. L. Marcus, G. Nieri, S. S. Philip, and J. D. Klausner, "Rectal gonorrhea and chlamydia reinfection is associated with increased risk of HIV seroconversion," Journal of Acquired Immune Deficiency Syndromes, vol. 53, no. 4, pp. 537-543, 2010.

[38] D. T. Fleming and J. N. Wasserheit, "From epidemiological synergy to public health policy and practice: the contribution 
of other sexually transmitted diseases to sexual transmission of HIV infection," Sexually Transmitted Infections, vol. 75, no. 1, pp. 3-17, 1999.

[39] C. T. Kane, S. Diawara, H. D. Ndiaye et al., "Concentrated and linked epidemics of both HSV-2 and HIV-1/HIV-2 infections in Senegal: public health impacts of the spread of HIV," International Journal of STD and AIDS, vol. 20, no. 11, pp. 793-796, 2009.

[40] S. C. Shafir, F. J. Sorvillo, and L. Smith, "Current issues and considerations regarding trichomoniasis and human immunodeficiency virus in African-Americans," Clinical Microbiology Reviews, vol. 22, no. 1, pp. 37-45, 2009.

[41] P. G. Larsson, M. Bergström, U. Forsum, B. Jacobsson, A. Strand, and P. Wölner-Hanssen, "Bacterial vaginosis transmission, role in genital tract infection and pregnancy outcome: an enigma," APMIS, vol. 113, no. 4, pp. 233-245, 2005.

[42] L. Westrom, G. Evaldson, and K. K. Holmes, "Taxonomy of vaginosis: bacterial vaginosis—a definition," Scandinavian Journal of Urology and Nephrology Supplement, vol. 86, pp. 259-264, 1984.

[43] J. Atashili, C. Poole, P. M. Ndumbe, A. A. Adimora, and J. S. Smith, "Bacterial vaginosis and HIV acquisition: a metaanalysis of published studies," AIDS, vol. 22, no. 12, pp. 14931501, 2008.

[44] U. Forsum, A. Hallén, and P. G. Larsson, "Bacterial vaginosis-a laboratory and clinical diagnostics enigma: review article II," APMIS, vol. 113, no. 3, pp. 153-161, 2005.

[45] U. Forsum, E. Holst, P. G. Larsson, A. Vasquez, T. Jakobsson, and I. Mattsby-Baltzer, "Bacterial vaginosis—a microbiological and immunological enigma," APMIS, vol. 113, no. 2, pp. 81-90, 2005.

[46] R. Amsel, P. A. Totten, and C. A. Spiegel, "Nonspecific vaginitis. Diagnostic criteria and microbial and epidemiologic associations," American Journal of Medicine, vol. 74, no. 1, pp. 14-22, 1983.

[47] R. P. Nugent, M. A. Krohn, and S. L. Hillier, "Reliability of diagnosing bacterial vaginosis is improved by a standardized method of gram stain interpretation," Journal of Clinical Microbiology, vol. 29, no. 2, pp. 297-301, 1991.

[48] P. Thorsen, I. P. Jensen, B. Jeune et al., "Few microorganisms associated with bacterial vaginosis may constitute the pathologic core: a population-based microbiologic study among 3596 pregnant women," American Journal of Obstetrics and Gynecology, vol. 178, no. 3, pp. 580-587, 1998.

[49] E. J. Huth, "Style notes: bacterial vaginosis or vaginal bacteriosis?" Annals of Internal Medicine, vol. 111, no. 7, pp. 553-554, 1989.

[50] P. Losikoff, R. Fichorova, B. Snyder et al., "Genital tract interleukin- 8 but not interleukin- $1 \beta$ or interleukin- 6 concentration is associated with bacterial vaginosis and its clearance in HIV-infected and HIV-uninfected women," Infectious Diseases in Obstetrics and Gynecology, vol. 2007, Article ID 92307, p. 6, 2007.

[51] D. Mares, J. A. Simoes, R. M. Novak, and G. T. Spear, “TLR2mediated cell stimulation in bacterial vaginosis," Journal of Reproductive Immunology, vol. 77, no. 1, pp. 91-99, 2008.

[52] C. M. Mitchell, J. Balkus, K. J. Agnew et al., "Bacterial vaginosis, not HIV, is primarily responsible for increased vaginal concentrations of proinflammatory cytokines," AIDS Research and Human Retroviruses, vol. 24, no. 5, pp. 667-671, 2008.

[53] A. M. Roberton, R. Wiggins, P. J. Horner et al., "A novel bacterial mucinase, glycosulfatase, is associated with bacterial vaginosis," Journal of Clinical Microbiology, vol. 43, no. 11, pp. 5504-5508, 2005.

[54] V. Pybus and A. B. Onderdonk, "Evidence for a commensal, symbiotic relationship between Gardnerella vaginalis and Prevotella bivia involving ammonia: potential significance for bacterial vaginosis," Journal of Infectious Diseases, vol. 175, no. 2, pp. 406-413, 1997.

[55] S. Cauci, S. Guaschino, S. Driussi, D. De Santo, P. Lanzafame, and F. Quadrifoglio, "Correlation of local interleukin-8 with immunoglobulin A against Gardnerella vaginalis hemolysin and with prolidase and sialidase levels in women with bacterial vaginosis," Journal of Infectious Diseases, vol. 185, no. 11, pp. 1614-1620, 2002.

[56] P. Hay, "Life in the littoral zone: lactobacilli losing the plot," Sexually Transmitted Infections, vol. 81, no. 2, pp. 100-102, 2005.

[57] K. C. Anukam, E. O. Osazuwa, I. Ahonkhai, and G. Reid, "Lactobacillus vaginal microbiota of women attending a reproductive health care service in Benin City, Nigeria," Sexually Transmitted Diseases, vol. 33, no. 1, pp. 59-62, 2006.

[58] S. L. Hillier, "The vaginal microbial ecosystem and resistance to HIV," AIDS Research and Human Retroviruses, vol. 14, supplement 1, pp. S17-S21, 1998.

[59] E. R. Boskey, R. A. Cone, K. J. Whaley, and T. R. Moench, "Origins of vaginal acidity: high D/L lactate ratio is consistent with bacteria being the primary source," Human Reproduction, vol. 16, no. 9, pp. 1809-1813, 2001.

[60] E. R. Boskey, K. M. Telsch, K. J. Whaley, T. R. Moench, and R. A. Cone, "Acid production by vaginal flora in vitro is consistent with the rate and extent of vaginal acidification," Infection and Immunity, vol. 67, no. 10, pp. 5170-5175, 1999.

[61] P. Daniel Johannes Rönnqvist, U. Birgitta Forsgren-Brusk, and E. Elisabeth Grahn-Håkansson, "Lactobacilli in the female genital tract in relation to other genital microbes and vaginal pH," Acta Obstetricia et Gynecologica Scandinavica, vol. 85, no. 6, pp. 726-735, 2006.

[62] S. J. Klebanoff and R. W. Coombs, "Viricidal effect of Lactobacillus acidophilus on human immunodeficiency virus type 1: possible role in heterosexual transmission," Journal of Experimental Medicine, vol. 174, no. 1, pp. 289-292, 1991.

[63] E. A. Fontaine and D. Taylor-Robinson, "Comparison of quantitative and qualitative methods of detecting hydrogen peroxide produced by human vaginal strains of lactobacilli," Journal of Applied Bacteriology, vol. 69, no. 3, pp. 326-331, 1990.

[64] L. Tao, S. Pavlova, J. Anzinger et al., "Fighting HIV with its natural enemy: mannose-specific capture of HIV by Lactobacillus," Beneficial Microbes Conference, Poster Abstract \#5B, 2005, http://www.asm.org/.

[65] V. Buffa, D. Stieh, N. Mamhood, Q. Hu, P. Fletcher, and R. J. Shattock, "Cyanovirin-N potently inhibits human immunodeficiency virus type 1 infection in cellular and cervical explant models," Journal of General Virology, vol. 90, no. 1, pp. 234243, 2009 .

[66] B. R. O’Keefe, F. Vojdani, V. Buffa et al., "Scaleable manufacture of HIV-1 entry inhibitor griffithsin and validation of its safety and efficacy as a topical microbicide component," Proceedings of the National Academy of Sciences of the United States of America, vol. 106, no. 15, pp. 6099-6104, 2009.

[67] J. M. Marrazzo, H. C. Wiesenfeld, P. J. Murray et al., "Risk factors for cervicitis among women with bacterial vaginosis," Journal of Infectious Diseases, vol. 193, no. 5, pp. 617-624, 2006. 
[68] H. Braat, J. van den Brande, E. Van Tol, D. Hommes, M. Peppelenbosch, and S. Van Deventer, "Lactobacillus rhamnosus induces peripheral hyporesponsiveness in stimulated CD4+ T cells via modulation of dendritic cell function," American Journal of Clinical Nutrition, vol. 80, no. 6, pp. 1618-1625, 2004.

[69] P. W. Lin, L. E. S. Myers, L. Ray et al., "Lactobacillus rhamnosus blocks inflammatory signaling in vivo via reactive oxygen species generation," Free Radical Biology and Medicine, vol. 47, no. 8, pp. 1205-1211, 2009.

[70] M. T. Tien, S. E. Girardin, B. Regnault et al., "Antiinflammatory effect of Lactobacillus casei on Shigella-infected human intestinal epithelial cells," Journal of Immunology, vol. 176, no. 2, pp. 1228-1237, 2006.

[71] J. J. Schellenberg, C. M. Card, T. B. Ball et al., "Bacterial vaginosis, HIV serostatus and T-cell subset distribution in a cohort of East African commercial sex workers: retrospective analysis," AIDS, vol. 26, no. 3, pp. 387-393, 2012.

[72] J. J. Schellenberg, M. G. Links, J. E. Hill et al., "Molecular definition of vaginal microbiota in east african commercial sex workers," Applied and Environmental Microbiology, vol. 77, no. 12, pp. 4066-4074, 2011.

[73] R. Hummelen, A. P. Vos, B. Van'T Land, K. Van Norren, and G. Reid, "Altered host-microbe interaction in HIV: a target for intervention with pro- and prebiotics," International Reviews of Immunology, vol. 29, no. 5, pp. 485-513, 2010.

[74] I. McGowan, "Microbicides for HIV prevention: reality or hope?" Current Opinion in Infectious Diseases, vol. 23, no. 1, pp. 26-31, 2010. 


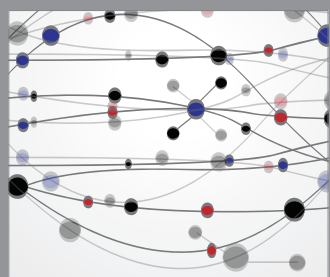

The Scientific World Journal
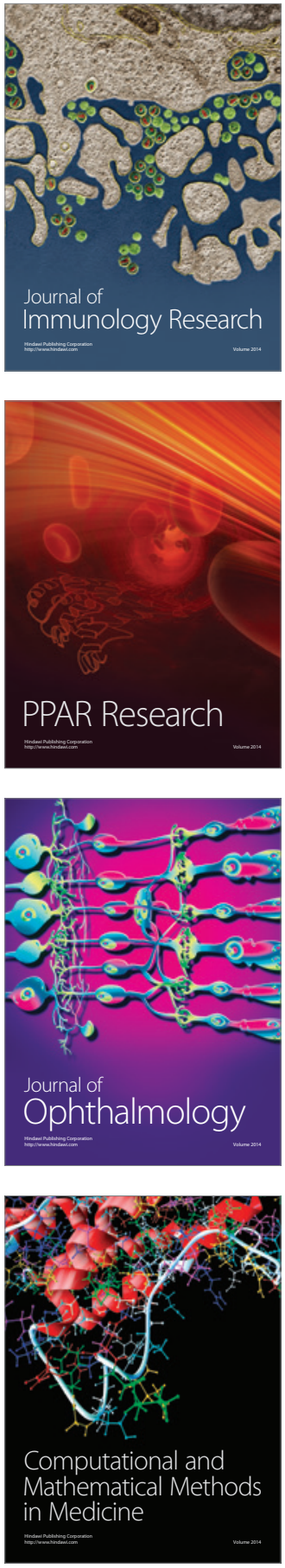

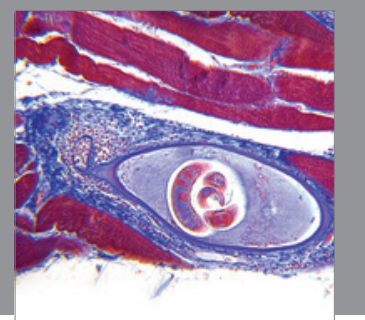

Gastroenterology

Research and Practice
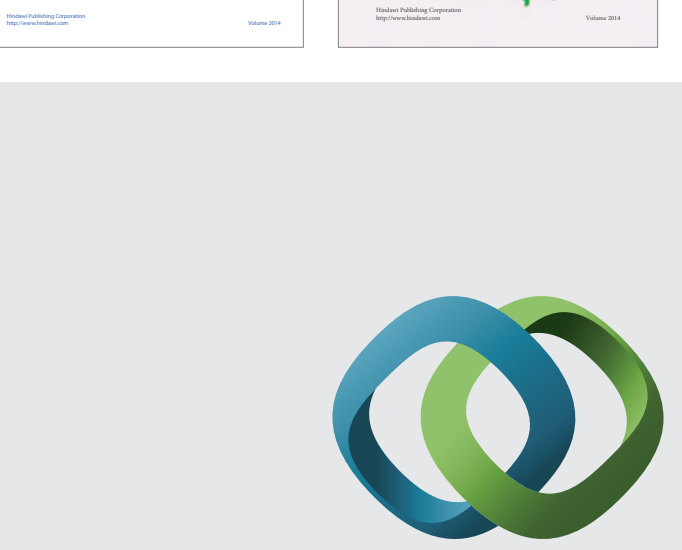

\section{Hindawi}

Submit your manuscripts at

http://www.hindawi.com
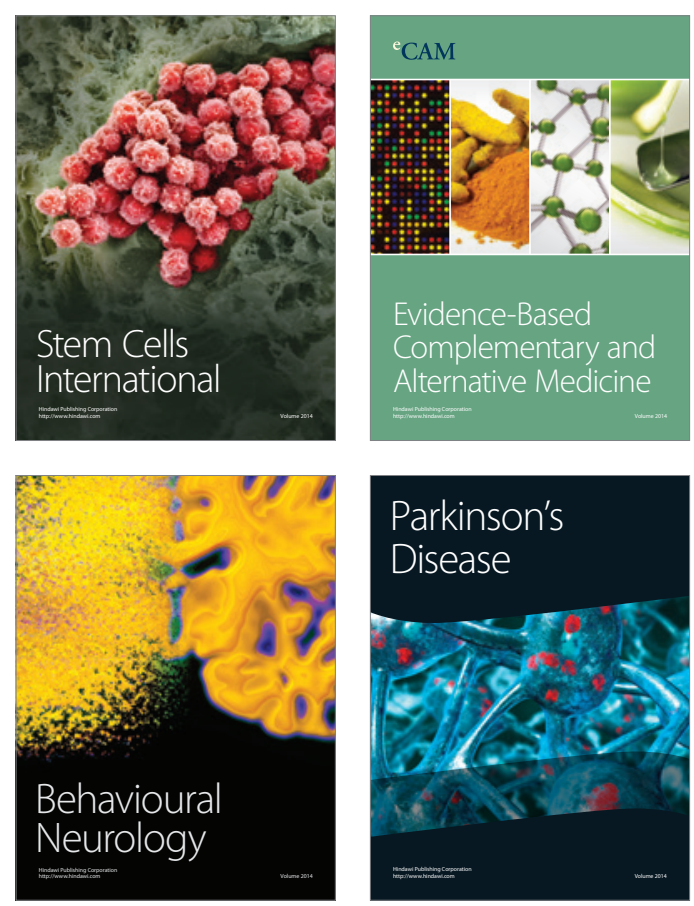

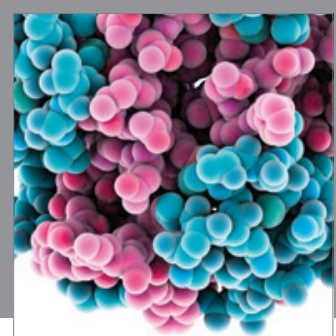

Journal of
Diabetes Research

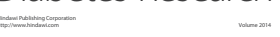

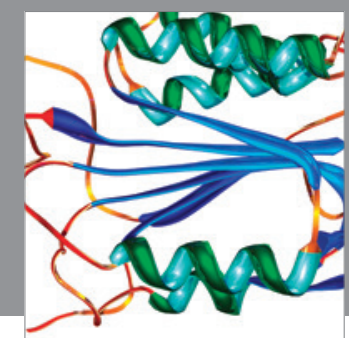

Disease Markers
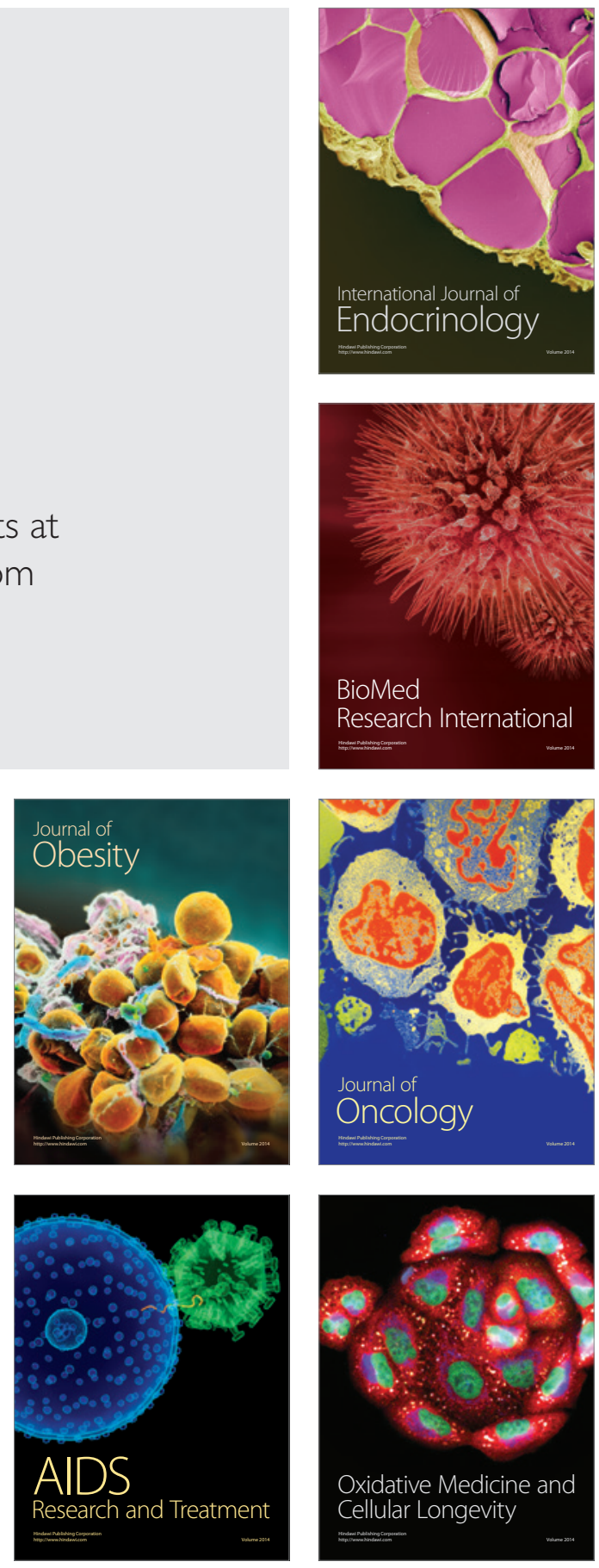